\title{
Spread and Control of Tuberculosis in the Case of Chiro Town: A Mathematical Model Analysis
}

\author{
Mume Yusuf Haso ${ }^{*}$, Dr.Temesgen Tibebu ${ }^{2}$ \\ ${ }^{1}$ Raya University, Ethiopia. \\ ${ }^{2}$ Associate Professor of Mathematical Modeling,Ethiopia.
}

*Corresponding Author: Mume Yusuf Haso, Raya University, Ethiopia.

\begin{abstract}
In this work, will be studied tuberculosis transmission using Susceptible-Exposed-InfectedCarrier-Recovered-Susceptible (SEIRS) Mathematical model analysis on the spread and control of tuberculosis of Chiro town population. We found that the dynamical system exhibit two equilibrium points (EPs) namely, disease free equilibrium point (DFEP) and endemic equilibrium point (EEP). Their stabilities were analyzed by the Routh-Hurwitz stability criterion. Thus we found that the DFEP is stable and the EEP is unstable. We also found that the basic reproduction number of the dynamical system is $R_{0}=\frac{p k \beta B}{\mu(k+\mu)(\mu+d+\gamma)}$ which depends on seven parameters. Using the real data collected from study area's population the numerical value of reproduction number is $R_{0}=0.4964$. That is $R_{0}<1$ which implies that the TB disease is not spread. Numerical simulations are performed to validate the theoretical results of our study. The control parameter that can help us to control the spread of the TB disease is transmission rate $\beta$, with numerical value $\beta=0.4732$. To control the spread of the TB disease and possibly eradicate the disease from the community, the transmission rate needs to be less than 0.4732 .
\end{abstract}

Keywords: dynamical system, Routh-Hurwitz stability criterion, reproduction number, Horizontal transmission, vertical transmission, compartment

Abbreviations: Tuberculosis (TB), Mycobacterium Tuberculosis (MTB), Latent Tuberculosis Infectious (LTBI), World Health Organization (WHO), Equilibrium point (EP), Disease free equilibrium point (DFEP), Endemic equilibrium point (EEP)

\section{INTRODUCTION}

Infection diseases are major cause of concern world-wide as they affect both human and animals. TB is also one of the infection disease in the world and the second most fatalistic human killer disease after HIV/AIDS, in the developing countries and as per current available data it causes millions of death every year [20]. Tuberculosis (TB) is an infection disease caused by mycobacterium tuberculosis, which is transmitted from an infected person to a susceptible person in airborne particles called droplet nuclei.

The TB bacteria can spread in the air from a person with active TB disease to others when they are in close contact. It is able to spread quickly through the air medium and so it is primarily transmitted through the respiratory route. When people who are infected with the disease are cough, sneeze, spit or talk, they propel TB germs (in mucus droplets), known as bacilli in to the air. A single sneeze can release up to 40,000 droplets. Each one of these droplets may transmit the disease since the infectious dose of tuberculosis is very low. Transmission occurs when a person inhales droplet nuclei containing tuberculosis bacteria. A previously uninfected person needs only to inhale a small number of these germs to be infected [5].

The TB disease is causes of few facilitates that they can be neglected, and is horizontally as well as vertically transmitted. Horizontal transmission is the passing of infection through some direct or indirect contact with infected individuals, for example, malaria and tuberculosis are horizontally transmitted diseases. Vertical transmission is the passing of infection from patients to new borne offspring, for example, AIDS, Chaga, and Hepatitis B are vertically (as well as horizontally) transmitted diseases[4]. 
The probability of transmission from one person to another depends up on several factors, including the number of infectious droplets expelled by the carrier, the effectiveness of ventilation, the duration of exposure, the virulence of the mycobacterium tuberculosis strains, the level of immunity in the un infected person, and others.

Different mathematical models have been developed incorporating some factors, such as fast and slow progression, drug-resistant stains, co-infection with HIV, relapse, re infection, migration, and vaccination to study the transmission dynamics of TB. These models are described by system of differential equations. Mathematical models have been used to improve our understanding of the basic transmission dynamics of $\mathrm{TB}$ and to evaluate the effectiveness of various control and prevention strategies $[2,15]$.

Data availability and the study objectives generally determine the modeling approach to be used. Accordingly, the deterministic models also known as the compartmental models are the main concerns of this study. Compartmental model, in which a population is divided in to sub-populations on the basis of such characteristics as TB status, has historically been the most common form of TB mathematical model. Compartmental models have been influential in modeling transmission dynamics of numerous infectious diseases, including droplet-borne respiratory diseases, sexually transmitted infections, and vector-borne diseases. The SIR, SEIR, SEIRS, SIS, and SI models, where $\mathrm{S}$ is susceptible, $\mathrm{E}$ is exposed $\mathrm{I}$ is infected, and $\mathrm{R}$ is recovered are the most commonly used compartmental models which are governed by nonlinear system of ODE that model TB transmission dynamics [24].

This paper is concerned with the analysis of the non-linear mathematical model studied using the SEICRS model of the spread and control of tuberculosis in the case of chiro town, west Haraghe, Oromia region, Ethiopia.. In this study the researcher wanted to extend the SEICRS model, where $\mathrm{S}$ is susceptible, $\mathrm{E}$ is exposed, I is infected, $\mathrm{C}$ is carrier, and $\mathrm{R}$ is recovered individuals; by assuming all compartments have equal natural death rate except infected compartment, infected compartment have death induced death rate.

\section{MeTHOdOLOGY}

In this study, the TB transmission dynamics between the compartments shall be described by a system of differential equation which shall be solved to obtain both the disease-free equilibrium point and the endemic equilibrium point. The stability analysis of those equilibrium points shall be carried out by using Routh-Hurwitz stability criteria. In this thesis our models used ordinary differential equation and analyses by determining and classifying their steady states.

\subsection{Basic Reproduction Number}

Basic reproduction number is measure of the potential for disease spread with in a population. The biological meaning of the reproduction number is the average number of secondary infections produced from one primary infected individual introduced in to a completely susceptible population. The basic reproduction number is denoted by $R_{0}$ and if $R_{0}<1$, a few infected individuals introduced in to completely susceptible population that means each individual produces on average less than one new infected individual and hence the disease dies out. On the other hand, if $R_{0}>1$, each individual produces on average more than one new infected individual and hence the disease is able to be invading the susceptible population [17]. If the $R_{0}=1$ the disease is remain in the population at the constant rate.

\subsection{Mathematical Model}

Mathematical models have been used to study the dynamics of infectious disease from more than a century. In recent years applications of Mathematics in infectious disease have shown remarkably growing trends. The earliest Mathematical model can be traced back to the $18^{\text {th }}$ century when Daniel Bernoulli formulated a model for small pox to estimate the effectiveness of variolation of healthy population with small pox. However, Mathematical models have been growing since the middle of the $20^{\text {th }}$ century after Kermack and Kendrick published their paper on epidemic models in 1927 which contains threshold results that determines whether an epidemic out break may occur or not[14].

\section{The model}

Under this we analyze the non-linear system of ODE of tuberculosis that describes our considered model SEICRS. In this model the total population is divided on to five compartments according to disease-state: 
These compartments are susceptible class $(\boldsymbol{S})$; un infected population but it can be infected for the future, Exposed class $(\boldsymbol{E})$; people who have come into contact with the disease but are not yet infective, Infected class $(\boldsymbol{I})$; those who have become infected with TB and are able to transmit the disease, Carrier class $(\boldsymbol{C})$; people after treatment having infection without showing symptoms; that is depending on natural immunity the treatment of some persons can take more time to be recovery than others then those population go to carrier class and $\operatorname{Recovered}$ class $(\boldsymbol{R})$; people who have recovered from the disease after treatment.

\section{Model assumptions}

The assumptions are:

- All recruitments in the total population are susceptible.

- There is no vertical transmission (all new births are susceptible).

- All compartments has equal natural death rate and there exists TB mortality rate of the infectious population.

- All parameters are positive.

- The latently infected individuals are considered as exposed to become TB infectious.

- The population is heterogeneously mixing with no restriction of age, sex, occupation and religion.

- An individual may infected only through contacts with infectious individuals.

- Successfully treated individuals can become recovered and un-Successfully treated individuals can become carrier again.

- The carrier individuals can also become recovered.

The flow chart and the corresponding dynamical system of the SEICRS model are as follow:

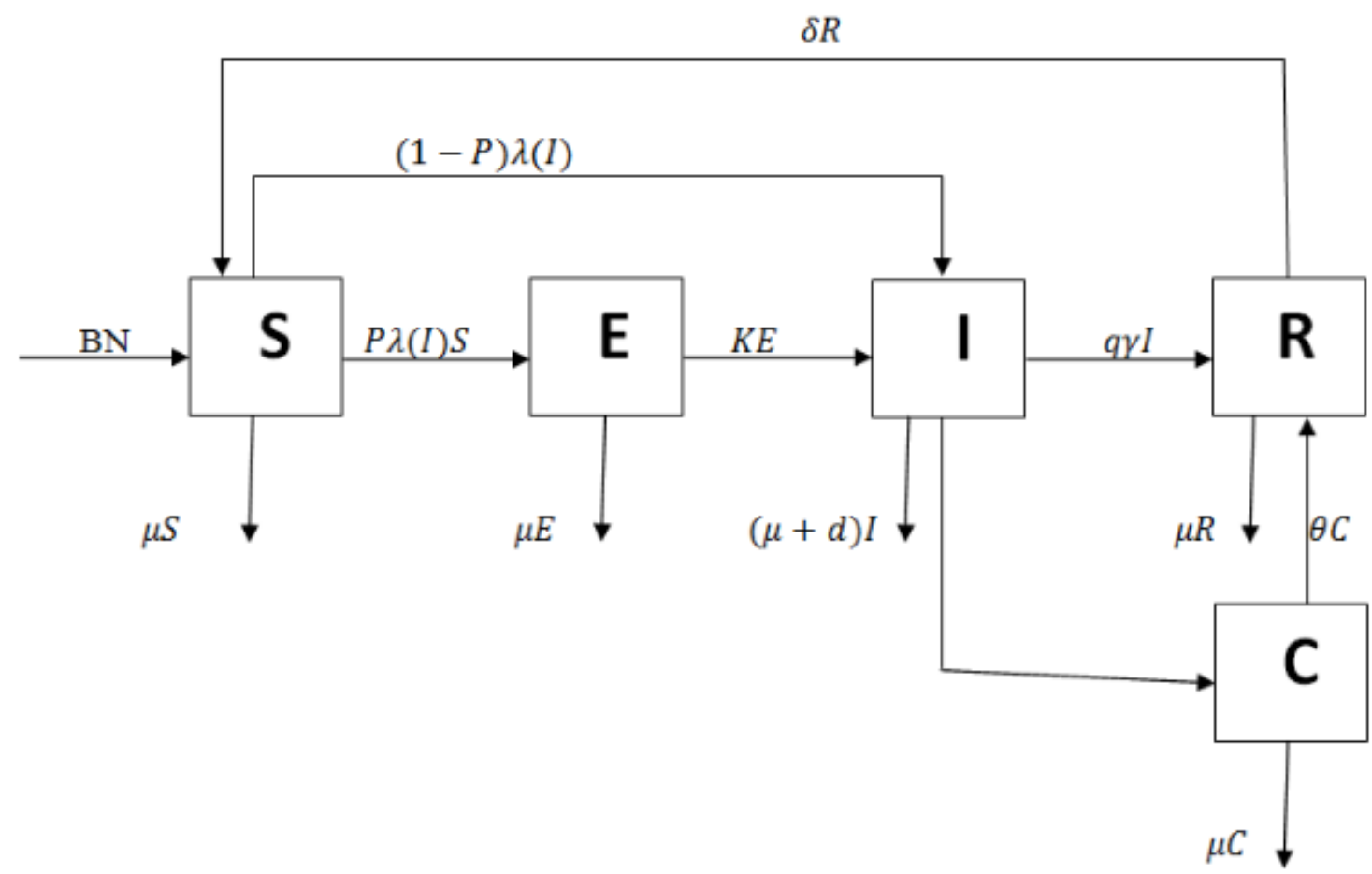

$$
\begin{aligned}
& \frac{d S}{d t}=B N+\delta R-\lambda(I) S-\mu S \\
& \frac{d E}{d t}=P \lambda(I) S-(k+\mu) E \\
& \frac{d I}{d t}=(1-P) \lambda(I) S+k E-(d+\gamma+\mu) I
\end{aligned}
$$


$\frac{d C}{d t}=(1-q) \gamma I-(\mu+\theta) C$

$\frac{d R}{d t}=q \gamma I-(\delta+\mu+\theta) R$

\section{State of Parameters and their Description}

Table1. state of parameters and their description

\begin{tabular}{|l|l|l|}
\hline Parameters & Formula & Description \\
\hline$d$ & $d=\frac{\text { number of individual who died by TB }}{\text { infected population }}$ & $\begin{array}{l}\text { Death induced death rat/e of } \\
\text { infected population }\end{array}$ \\
\hline$\delta$ & $\delta=\frac{\text { the number of people who again entered in to susceptible }}{\text { the total number of recovered population }}$ & Re-susceptibility rate \\
\hline$\mu$ & $\gamma=\frac{1}{\text { infectious period }}$ & Recovery or removal rate \\
\hline$k$ & $\mu=\frac{\text { number of individual who died by natural reason }}{\text { total number of population }}$ & Natural death rate \\
\hline$\beta$ & $k=\frac{1}{\text { incubation period }}$ & Infectious rate \\
\hline$\theta$ & $\beta=\frac{\text { number of TB infectious }}{\text { number of people who took TB test }}$ & Transmission rate \\
\hline$B$ & $\theta=\frac{\text { the number of carrier population who become recovered }}{\text { the total number of carrier population }}$ & $\begin{array}{l}\text { The rate at which carrier } \\
\text { population goes to recovered }\end{array}$ \\
\hline$\lambda(I)$ & $B=\frac{\text { total number of new born babies from total population }}{\text { total number of population }}$ & The recruitment rate \\
\hline
\end{tabular}

In our model, the total population $\mathrm{N}$ is divided in to five compartments: susceptible, exposed, infected, carrier and recovered, with their densities denoted by $S(t), E(t), I(t), C(t)$ and $R(t)$ respectively. That is $N(t)=S(t)+E(t)+I(t)+C(t)+R(t)$. The rates of these compartments are represented by the dynamical system (1) with initial conditions $S(0)>0 E(0)>0, I(0)>0, C(0)>$ 0 , and $R(0)>0$. We represent the proportion of the compartments $S(t), E(t), I(t), C(t)$ and $R(t)$ by:

$$
s(t)+e(t)+i(t)+c(t)+c(t)=\frac{S(t)+E(t)+I(t)+C(t)+R(t)}{N(t)}=\frac{N(t)}{N(t)}=1
$$

Thus $s(t)=\frac{S(t)}{N(t)}$ implies that $\frac{d s}{d t}=\frac{1}{N^{2}}\left(N \frac{d s}{d t}-S \frac{d N}{d t}\right)=\frac{1}{N} \frac{d S}{d t}$

$e(t)=\frac{E(t)}{N(t)}$ Implies that $\frac{d e}{d t}=\frac{1}{N^{2}}\left(N \frac{d E}{d t}-E \frac{d N}{d t}\right)=\frac{1}{N} \frac{d E}{d t}$

$i(t)=\frac{I(t)}{N(t)}$ Implies that $\frac{d i}{d t}=\frac{1}{N^{2}}\left(N \frac{d I}{d t}-I \frac{d N}{d t}\right)=\frac{1}{N} \frac{d I}{d t}$

$c(t)=\frac{C(t)}{N(t)}$ Implies that $\frac{d c}{d t}=\frac{1}{N^{2}}\left(N \frac{d C}{d t}-C \frac{d N}{d t}\right)=\frac{1}{N} \frac{d C}{d t}$

$r\left(t^{\prime}\right)=\frac{S(t)}{N(t)}$ Implies that $\frac{d r}{d t}=\frac{1}{N^{2}}\left(N \frac{d R}{d t}-R \frac{d N}{d t}\right)=\frac{1}{N} \frac{d R}{d t}$

Here, in all of the five cases $\mathrm{N}$ is constant and $\frac{d N}{d t}=0$.

$$
\begin{aligned}
& \frac{d s}{d t}=\frac{1}{N}\left[\frac{d S}{d t}\right]=\frac{1}{N}[B N+\delta R-\lambda(I) S-\mu S]=B+\delta r-\beta s i-\mu s \\
& \frac{d e}{d t}=\frac{1}{N}\left[\frac{d E}{d t}\right]=\frac{1}{N}[P \lambda(I) S-(K+\mu) E]=p_{\beta} s i-(K+\mu) e \\
& \frac{d i}{d t}=\frac{1}{N}\left[\frac{d I}{d t}\right]=\frac{1}{N}[(1-P) \lambda(I) S+K E-(d+\gamma+\mu) I]=(1-p) \beta s i+K e-(d+\gamma+\mu) i \\
& \frac{d c}{d t}=\frac{1}{N}\left[\frac{d C}{d t}\right]=\frac{1}{N}[(1-q) \gamma I-(\mu+\theta) C]=(1-q) \gamma i-(\mu+\theta) c \\
& \frac{d r}{d t}=\frac{1}{N}\left[\frac{d I}{d t}\right]=\frac{1}{N}[q \gamma I-(\delta+\mu+\theta) R]=q \gamma i-(\delta+\mu+\theta) r
\end{aligned}
$$

The new dynamical system which is equivalent to the dynamical system (1)-(5) is:

$\frac{d s}{d t}=B+\delta r-\beta^{s i}-\mu s$ 


$$
\begin{aligned}
& \frac{d e}{d t}=p_{\beta} s i-(K+\mu) e \\
& \frac{d i}{d t}=(1-p) \beta_{s i}+K e-(d+\gamma+\mu) i \\
& \frac{d c}{d t}=(1-q) \gamma i-(\mu+\theta) c \\
& \frac{d r}{d t}=q \gamma i-(\delta+\mu+\theta) r
\end{aligned}
$$

\subsection{Model Analysis}

\section{Equilibrium Points Basic Reproduction Number of the Model}

The system (2) has two equilibrium points: Disease free equilibrium $E\left(\frac{B}{\mu}, 0,0,0,0\right)$ and Endemic equilibrium point

$$
\begin{aligned}
& E^{*}\left(s^{*}, e^{*}, i^{*}, c^{*}, r^{*}\right) \\
& =\left(\begin{array}{c}
\frac{p(k+\mu)(\mu+d+\gamma)}{\beta(k+(1-p) \mu)}, \frac{p(\mu+d+\gamma)(\delta+\mu+\theta)[\mu(k+\mu)(\mu+d+\gamma)-\beta B(k+(1-p) \mu)]}{\beta(k+(1-p) \mu)[\delta q \gamma(k+(1-p) \mu)-(k+\mu)(\delta+\mu+\theta)(\mu+d+\gamma)}, \\
\frac{(\delta+\mu+\theta)[\mu(k+\mu)(\mu+d+\gamma)-\beta B(k+(1-p) \mu)]}{\beta[\delta q \gamma(k+(1-p) \mu)-(k+\mu)(\delta+\mu+\theta)(\mu+d+\gamma)]}, \\
\frac{(1-q) \gamma(\delta+\mu+\theta)[\mu(k+\mu)(\mu+d+\gamma)-\beta B(k+(1-p) \mu)]}{(\mu+\theta) \beta[\delta q \gamma(k+(1-p) \mu)-(k+\mu)(\delta+\mu+\theta)(\mu+d+\gamma)]}, \\
\frac{q \gamma(\delta+\mu+\theta)[\mu(k+\mu)(\mu+d+\gamma)-\beta B(k+(1-p) \mu)]}{\beta(\delta+\mu+\theta)[\delta q \gamma(k+(1-p) \mu)-(k+\mu)(\delta+\mu+\theta)(\mu+d+\gamma)]}
\end{array}\right)
\end{aligned}
$$

Satisfying the system:

$$
\left\{\begin{array}{c}
B+\delta r-\beta s i-\mu s=0 \\
p_{\beta} s i-(K+\mu) e=0 \\
(1-p) \beta s i+K e-(d+\gamma+\mu) i=0 \\
(1-q) \gamma i-(\mu+\theta) c=0 \\
q \gamma i-(\delta+\mu+\theta) r=0
\end{array}\right.
$$

Proposition: The reproduction number of our model is $R_{0}=\frac{p k \beta B}{\mu(k+\mu)(\mu+d+\gamma)}$

Proof: To determine the reproduction number of the dynamical system (2) by using next generation method, we need to rearrange the dynamical system (2) as the rates of change of new TB infection present are $\frac{d e}{d t}, \frac{d i}{d t}$ and $\frac{d c}{d t}$.

The rearranged dynamical system is as follows:

$$
\begin{aligned}
& \frac{d e}{d t}=p_{\beta} s i-(K+\mu) e \\
& \frac{d i}{d t}=(1-p)_{\beta s i}+K e-(d+\gamma+\mu) i \\
& \frac{d c}{d t}=(1-q) \gamma i-(\mu+\theta) c \\
& \frac{d s}{d t}=B+\delta r-\beta s i-\mu s \\
& \frac{d r}{d t}=q \gamma i-(\delta+\mu+\theta) r
\end{aligned}
$$

The rate of change of new infections in the dynamical system (3) is:

$$
f(e, i, c, s, r)=\left(\begin{array}{c}
\beta s i \\
0 \\
0 \\
0 \\
0
\end{array}\right)
$$

The rate of transfer of individuals into each compartment is: 
$V^{+}(e, i, c, s, r)=\left(\begin{array}{c}0 \\ k e \\ (1-q) \gamma i \\ B+\delta r \\ q \gamma i\end{array}\right)$ And

The rate of transfer of individuals into each compartment is:

$$
V^{-}(e, i, c, s, r)=\left(\begin{array}{c}
(k+\mu) e \\
(\mu+d+\gamma) i \\
(\mu+\theta) c \\
(\beta i+\mu) s \\
(\delta+\mu+\theta) r
\end{array}\right)
$$

The rate of transfer of individuals in and out of each compartment is:

$$
\begin{aligned}
V(e, i, c, s, r)= & V^{-}(e, i, c, s, r)-V^{+}(e, i, c, s, r)=\left(\begin{array}{c}
(k+\mu) e \\
(\mu+d+\gamma) i \\
(\mu+\theta) c \\
(\beta i+\mu) s \\
(\delta+\mu+\theta) r
\end{array}\right)-\left(\begin{array}{c}
0 \\
k e \\
(1-q) \gamma i \\
B+\delta r \\
q \gamma i
\end{array}\right) \\
= & \left(\begin{array}{c}
(k+\mu) e \\
-k e+(\mu+d+\gamma) i \\
-(1-q) \gamma+(\mu+\theta) c \\
-B-\delta r+(\beta i+\mu) s \\
-q \gamma i+(\delta+\mu+\theta) r
\end{array}\right)
\end{aligned}
$$

The Jacobean matrix of $f(e, i, c, s, r)$ and $V(e, i, c, s, r)$ at any equilibrium points are:

$$
\begin{aligned}
& D v(E)=\left(\begin{array}{ll}
V & 0 \\
J_{3} & J_{4}
\end{array}\right) \text { and } D f\left(E^{*}\right)=\left(\begin{array}{ll}
F & 0 \\
0 & 0
\end{array}\right) \quad F=\left(\begin{array}{ccc}
0 & \frac{P \beta B}{\mu} & 0 \\
0 & 0 & 0 \\
0 & 0 & 0
\end{array}\right) \\
& V=\left(\begin{array}{ccc}
k+\mu & 0 & 0 \\
-k & \mu+d+\gamma & 0 \\
0 & -(1-q) \gamma & \mu+\theta
\end{array}\right) \\
& F V^{-1}=\left(\begin{array}{ccc}
\frac{p k \beta B}{\mu(k+\mu)(\mu+d+\gamma)} & \frac{p \beta B}{\mu(\mu+d+\gamma)} & 0 \\
0 & 0 & 0 \\
0 & 0 & 0
\end{array}\right)
\end{aligned}
$$

The spectral radius is of $\mathrm{FV}^{-1}$ is obtained from

$$
\left|F V^{-1}-\lambda I\right|=0 \quad \lambda_{1}=\lambda_{2}=0 \text { or } \lambda_{3}=\frac{p k \beta B}{\mu(k+\mu)(\mu+d+\gamma)}
$$


Hence, the spectral radius of the next generation matrix is given by $\max \left\{\lambda_{1}, \lambda_{2}, \lambda_{3}\right\}=\lambda_{3}$.

Therefore $\lambda_{3}$ is the required reproduction number, then $R_{0}=\frac{p k \beta B}{\mu(k+\mu)(\mu+d+\gamma)}$.

\section{Parameter estimation}

The following parameters are taken from our real collected data: $\beta=0.4732, \mu=0.0011, d=$ $0.0219, B=0.0317, k=8.6905, \gamma=26.0714, \delta=0.5938, \theta=0.2049, \quad \mathrm{~B}=0.0317 \quad S(0)=$ $31121, E(0)=22643, I(0)=274, C(0)=204$ and $R(0)$.

The disease free equilibrium point is: $E(s, e, i, c, r)=(28.8181818,0,0,0,0)$ and the endemic equilibrium point is:

$E^{*}\left(s^{*}, e^{*}, i^{*}, c^{*}, r^{*}\right)=(52.39361971087,0.45073141775,0.00006641009,0.01516616897,0.05170015925)$

Based on the Routh-Hurwitz stability criteria the free disease equilibrium point is stable; this implies that the disease is not spread in the community and the endemic equilibrium point is unstable.

The reproduction number is becomes $R_{0}=0.49640098614$, here, the reproduction number is less than one. This shows that the disease free equilibrium point is stable and the endemic equilibrium point is unstable. This indicates that TB disease cannot be spread in the community of Chiro town and the disease will die out in the long run.

\section{NUMERICAL SimUlation}

In this section we consider the reproduction number of the extended model is given by $R_{0}=$ $\frac{p k \beta B}{\mu(k+\mu)(\mu+d+\gamma)}$, which depends on seven parameters where the parameters are given in table 1. In this section we are going to analysis the strong effect of each parameter change on the reproduction number.

1. The graphical representation of the reproduction number and versus the transmission rate is given by:

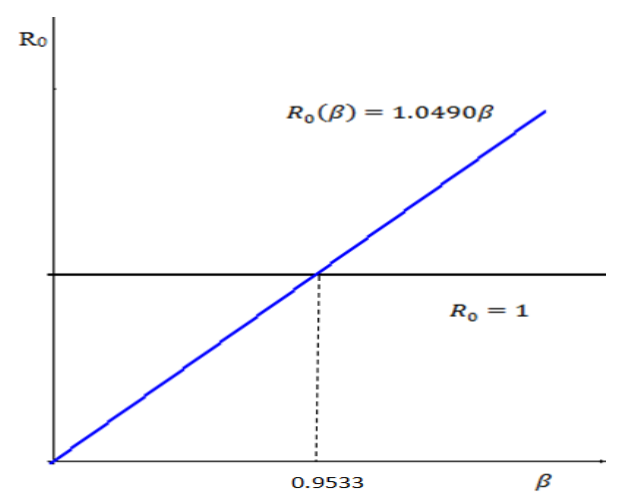

Figure1. The graph of $\beta$ versus $R_{0}$

2. The graphical representation of the reproduction number and versus the recruitment rate is given by:

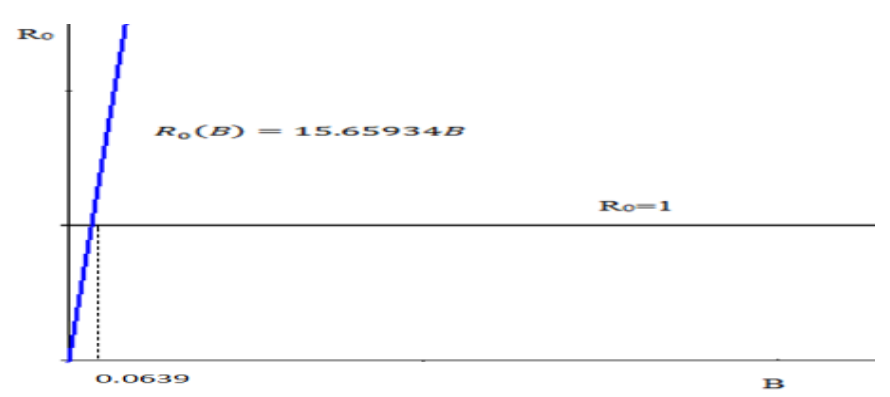

Figure2. The graph of $k$ versus $R_{0}$ 
3. The graphical representation of the reproduction number and versus the recovery rate is given by:

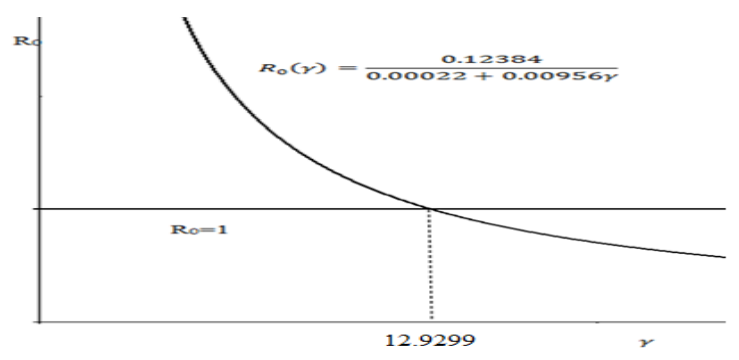

Figure3. The graph of $\gamma$ versus $R_{0}$

4. The graphical representation of the reproduction number and versus the proportion of fast progression is given by:

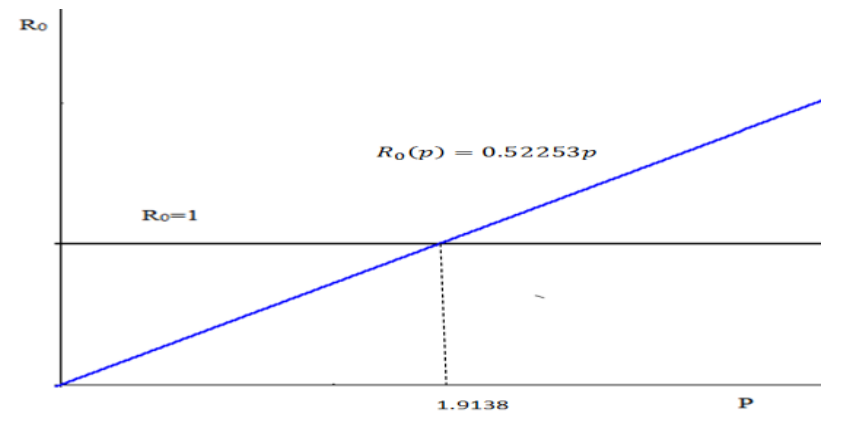

Figure4. The graph of $p$ versus $R_{0}$

NB: Parameters that are not discussed under this has no more effect on the reproduction number.

\section{DISCUSSION AND RESULTS}

In this thesis we attempt to study a mathematical model of the transmission dynamics of TB by considering the SEICRS model to predict the combined effect on the spread and control of tuberculosis in Chiro town. The SEICRS TB model was formulated using a deterministic a five dimensional system of differential equation to effectively investigate the transmission dynamics of TB and to find out the most effective the control measure.

A complete qualitative analysis of the model was done. It was first showed that the positivity of solution, where the model was epidemiologically meaningful and mathematically well posed and boundedness of the solution region. The model has two equilibrium points, namely the disease free equilibrium point and the endemic equilibrium point. By analyzing the model, we found a threshold parameter called the basic reproduction number which is $R_{0}=\frac{p k \beta B}{\mu(k+\mu)(\mu+d+\gamma)}$ and it was calculated by using the next generation matrix and it was established that the disease free equilibrium point is asymptotically stable when the reproduction number is less than one and the model is unstable when the reproduction number is greater than one.

The stability analysis of the model was investigated by using Routh-Hurwitz stability criteria. The SEICRS model has two non-negative equilibrium points, the disease free equilibrium point $(28.8181818,0,0,0,0)$, and the endemic equilibrium point

(52.39361971087,0.45073141775,0.00006641009,0.01516616897,0.05170015925). Using Routh-Hurwitz stability criteria, disease free equilibrium point is stable and the endemic equilibrium point is unstable.

From figure-1: when transmission rate increases $(\beta>0.9533)$, then the reproduction number $R_{0}>$ 1 , this means the disease persist. When the transmission rate decrease $(\beta<0.9533)$, then the reproduction number is $R_{0}<1$, this implies that the disease died out.

From figure-2: when natural birth rate increases $(B>0.0639)$, then the reproduction number $R_{0}>$ 1 , the disease persist. When the transmission rate decrease $\beta<0.0639$, then the reproduction number is $R_{0}<1$, this implies that the spread of the disease reduces. 
From figure-3: when the recovery rate $\gamma>12.9299$, then the reproduction number $R_{0}<1$, implies that the disease does not spread in society. When the recovery rate $\gamma<12.9299$, then the reproduction number $R_{0}>1$, this means the disease spread in society.

From figure-4: when proportion of fast progression increases ( $>1.9138)$, then the reproduction number $R_{0}>1$, this means the disease persist. When $\mathrm{p}<1.9138$ the reproduction number $R_{0}<1$, this means the disease died out.

\section{CONCLUSION}

In this thesis the researcher formulate the deterministic mathematical model on TB dynamics with fast progression and slow progression, and analysis the possible way of eradicating TB. From the model, it can be noticed that the spread of the disease largely depends on the transmission rate $\beta$ and the control of the disease also depends on the removal rate $\gamma$. The stability analysis of equilibrium points are investigated by Routh-Hurwitz stability criteria. From the stability analysis of equilibrium points and numerical simulation result, the researcher concluded that the disease does not spread through the community. From the results, the value of the basic reproduction number is small (less than one) from this we can conclude that the disease is not spread in Chiro town.

\section{ACKNOWLEDGMENTS}

First of all, I would like to grateful to the almighty Allah who has given me a life to live, health and wisdom to perform all the necessary tasks possible for write this thesis. Next, I am particularly grateful to my advisor Temesgen Tibebu (ph.D) for guiding and directing the success of this thesis.

I would thanks to my parents and family for guiding me by concerned idea to help me for this success. Finally, I would like to thanks all concerned bodies of governmental and private health institutions in Chiro town such as Chiro zonal hospital, Chiro town health center and Tesfa medium clinic for their cooperation to give me reliable and accurate data which helps to as in put for me.

\section{REFERENCES}

[1] A.U.Kalu ${ }^{1}$ and S.C.Inyama ${ }^{2}$, Mathematical model of the role of vaccination and treatment on the transmission dynamics of tuberculosis, Federal university of Technology, Owerri, Imo state,Nigeria,2012.

[2] HongbinGuoand Michael Y. Li, Global stability in a Mathematical model of tuberculosis, 2006.

[3] Juan puploAparicio, Carlos Castillo-chaves, Mathematical modeling of tuberculosis epidemics, 2009.

[4] Mohammed El-Doma, Analysis of an SIRS Age-structured epidemic model with vaccination and vertical transmission of disease, 2006.

[5] Taxance MBAYA, Jean Luc DIMI,Bienvenu ONDAMi, Mathematical analysis of deterministic and stochastic model of Tuberculosis, University marien Ngoubi, BP69Brazzavillecongo, 2016.

[6] Muhammad RifkiTaufik, Dwi Lestari, and Tri WijayantiSeptiarini, Mathematical model of vaccinated Tuberculosis disease, June 2015.

[7] M.O.Ibrahim,C.N.Ejieji,a,S.A.Egbetade b, a Maathematical model for the epidemiology of tuberculosis with estimate of the basic reproduction number, 2013.

[8] S.A.Egbetade ${ }^{1, *}$, M.O.Ibrahim2, on the existence of solution of A tuberculosis epidemic model, University of Ilorin, Nigeria, 2012.

[9] Samuel Bowng a, b, 1, JurgenKurths,b,c, Parameter estimation based synchronization for an epidemic model with applications to TB in Cameroon,2010.

[10] Zhilan Feng, Angel f. Copurro, A model for tuberculosis with exogenous re-infection, 2000.

[11] Dany Pascal Moualeu-Ngangue, a Mathematical tuberculosis model in Cameroon, May 2013.

[12] Luju Liu, ${ }^{\mathrm{a}, \mathrm{b},{ }^{*}, X i a o-Q i a n g} \mathrm{Zhao}^{\mathrm{b}}, \mathrm{Yicang} \mathrm{Zhou}^{\mathrm{a}}, \mathrm{A}$ tuberculosis model with seasonality, Xi'anjiaotong university, Xi'an710049, China, university of Newtoundland, NLAIC5S7,Canada, 2010.

[13] Emmanuel Appo H Andam, Analysis of transmission dynamics of tuberculosis using differential equations: A case study of Amansie West District, Ghana, 2013.

[14] AshrafuRahman, university of western Ontario, Study of infectious disease by Mathematical models: Predictions and controls, 2016.

[15] Embay Rohaeti, Sri Wardatum and AniAndriyati, Stability analysis model of spreading controlling of tuberculosis, 2015.

[16] Elizabeth L Carbott, Berbera Marston, Gavin J Chunchyard, Kevin M Decock, Tuberculosis in SubSaharan Africa, 2006. 
[17] P.van den Driessche ${ }^{1}$ and James watmough ${ }^{2}$, Further notes on the Basic Reproduction number, University of Victoria Canada, V8W3P4, University of New Brunswick Canada, E3B5A3.

[18] O.Dieckmann,J.A.P.Heesterbeck, J.A.J.Metz, On the definition and computation of the basic reproduction in model for infectious disease,J.Math.Biol.35:503[522,1990].

[19] Jain A, Mondal R: Extensively drug-resistant tuberculosis Current challenges and threats FEMS Immunol med microbial, 2008.

[20] World Health Organization -Global tuberculosis control, 2011.

[21] R.Shrestha-kuwahara, M.Wilke, HA.Joseph, JW.Carey, R.Plak, and E.Sumartojo, Tuberculosis Research and control Antropological contribution.

[22] Global tuberculosis control World Health Organization report, 2004.

[23] First Ethiopian national population based on tuberculosis prevalence survey, 2001.

[24] Hloniphile sithole, Mathematical analysis of tuberculosis vaccine models and control strategies, University of KwaZulu-Natal, 2014.

Citation: Mume Yusuf Haso \& Dr.Temesgen Tibebu (2020). Spread and Control of Tuberculosis in the Case of Chiro Town: A Mathematical Model Analysis. International Journal of Scientific and Innovative Mathematical Research (IJSIMR), 8(1), pp. 23-32. http://dx.doi.org/ 10.20431/2347 -3142.0801004

Copyright: (C) 2020 Authors, this is an open-access article distributed under the terms of the Creative Commons Attribution License, which permits unrestricted use, distribution, and reproduction in any medium, provided the original author and source are credited. 